International Journal of Pure and Applied Mathematics

Volume 86 No. 1 2013, 199-216

ISSN: $1311-8080$ (printed version); ISSN: 1314-3395 (on-line version)

url: http://www.ijpam.eu

doi: http://dx.doi.org/10.12732/ijpam.v86i1.13

ijpam.eu

\title{
REGULAR ELEMENTS OF THE COMPLETE SEMIGROUPS OF BINARY RELATIONS OF THE CLASS $\sum_{7}(X, 8)$
}

\author{
Barış Albayrak $^{1}$, I. Yasha Diasamidze ${ }^{2}$, Neşet Aydin ${ }^{3}$ \\ ${ }^{1,3}$ Department of Mathematics \\ Faculty of Science and Art \\ Canakkale Onsekiz Mart University \\ Canakkale, TURKEY \\ ${ }^{2}$ Shota Rustaveli State University \\ 35, Ninoshvili St., Batumi 6010, GEORGIA
}

\begin{abstract}
In this paper let $Q=\left\{T_{1}, T_{2}, T_{3}, T_{4}, T_{5}, T_{6}, T_{7}, T_{8}\right\}$ be a subsemilattice of $X$-semilattice of unions $D$ where $T_{1} \subset T_{2} \subset T_{3} \subset T_{5} \subset T_{6} \subset T_{8}$, $T_{1} \subset T_{2} \subset T_{3} \subset T_{5} \subset T_{7} \subset T_{8}, T_{1} \subset T_{2} \subset T_{4} \subset T_{5} \subset T_{6} \subset T_{8}, T_{1} \subset T_{2} \subset$ $T_{4} \subset T_{5} \subset T_{7} \subset T_{8}, T_{1} \neq \emptyset, T_{4} \backslash T_{3} \neq \emptyset, T_{3} \backslash T_{4} \neq \emptyset, T_{6} \backslash T_{7} \neq \emptyset, T_{7} \backslash T_{6} \neq \emptyset$, $T_{3} \cup T_{4}=T_{5}, T_{6} \cup T_{7}=T_{8}$, then we characterize the class each element of which is isomorphic to $Q$ by means of the characteristic family of sets, the characteristic mapping and the generate set of $Q$. Moreover, we calculate the number of regular elements of $B_{X}(D)$ for a finite set $X$.
\end{abstract}

AMS Subject Classification: 20M30, 20M10, 20M15

Key Words: semigroups, binary relation, regular elements

\section{Introduction}

Let $X$ be an arbitrary nonempty set. Recall that a binary relation on $X$ is a subset of the cartesian product $X \times X$. The binary operation $\circ$ on $B_{X}$ (the set 
of all binary relations on X) defined by for $\alpha, \beta \in B_{X}$

$$
(x, z) \in \alpha \circ \beta \Leftrightarrow(x, y) \in \alpha \text { and }(y, z) \in \beta \text {, for some } y \in X
$$

is associative. Therefore $B_{X}$ is a semigroup with respect to the operation $\circ$. This semigroup is called the semigroup of all binary relations on the set $X$.

Let $D$ be a nonempty set of subsets of $X$ which is closed under the union i.e., $\cup D^{\prime} \in D$ for any nonempty subset $D^{\prime}$ of $D$. In that case, $D$ is called a complete $X$ - semilattice of unions. The union of all elements of $D$ is denoted by the symbol $\breve{D}$. Clearly, $\breve{D}$ is the largest element of $D$.

Let $X$ be an arbitrary nonempty set and $m$ be an arbitrary cardinal number. $\Sigma(X, m)$ is the class of all complete $X$ - semilattices of unions of power $m$.

Let $\widetilde{D}$ and $D^{\prime}$ be some nonempty subsets of the complete $X$ - semilattices of unions. We say that a subset $\widetilde{D}$ generates a set $D^{\prime}$ if any element from $D^{\prime}$ is a set-theoretic union of the elements from $\widetilde{D}$.

Note that the semilattice $D$ is partially ordered with respect to the settheoretic inclusion. Let $\emptyset \neq D^{\prime} \subseteq D$ and

$$
N\left(D, D^{\prime}\right)=\left\{Z \in D \mid Z \subseteq Z^{\prime} \text { for any } Z^{\prime} \in D^{\prime}\right\} .
$$

It is clear that $N\left(D, D^{\prime}\right)$ is the set of all lower bounds of $D^{\prime}$. If $N\left(D, D^{\prime}\right) \neq \emptyset$ then $\Lambda\left(D, D^{\prime}\right)=\cup N\left(D, D^{\prime}\right)$ belongs to $D$ and it is the greatest lower bound of $D^{\prime}$.

Further, let $x, y \in X, Y \subseteq X, \alpha \in B_{X}, T \in D, \emptyset \neq D^{\prime} \subseteq D$ and $t \in \breve{D}$. Then we have the following notations,

$$
\begin{array}{ll}
y \alpha=\{x \in X \mid(y, x) \in \alpha\} & , Y \alpha=\bigcup_{y \in Y} y \alpha, \\
V(D, \alpha)=\{Y \alpha \mid Y \in D\} & , D_{t}=\left\{Z^{\prime} \in D \mid t \in Z^{\prime}\right\}, \\
D_{T}^{\prime}=\left\{Z^{\prime} \in D^{\prime} \mid T \subseteq Z^{\prime}\right\} & , \ddot{D}_{T}^{\prime}=\left\{Z^{\prime} \in D^{\prime} \mid Z^{\prime} \subseteq T\right\} .
\end{array}
$$

Let $f$ be an arbitrary mapping from $X$ into $D$. Then one can construct a binary relation $\alpha_{f}$ on $X$ by $\alpha_{f}=\bigcup_{x \in X}(\{x\} \times f(x))$. The set of all such binary relations is denoted by $B_{X}(D)$. It is easy to prove that $B_{X}(D)$ is a semigroup with respect to the operation $\circ$. In this case $B_{X}(D)$, is called a complete semigroup of binary relations defined by an $X$-semilattice of unions $D$. This structure was comprehensively investigated in Diasemidze [6].

If $\alpha \circ \beta \circ \alpha=\alpha$ for some $\beta \in B_{X}(D)$ then a binary relation $\alpha$ is called a regular element of $B_{X}(D)$. 
Let $\alpha \in B_{X}, Y_{T}^{\alpha}=\{y \in X \mid y \alpha=T\}$ and

$$
V[\alpha]=\left\{\begin{array}{l}
V\left(X^{*}, \alpha\right), \text { if } \emptyset \notin D, \\
V\left(X^{*}, \alpha\right), \text { if } \emptyset \in V\left(X^{*}, \alpha\right), \\
V\left(X^{*}, \alpha\right) \cup\{\emptyset\}, \text { if } \emptyset \notin V\left(X^{*}, \alpha\right) \text { and } \emptyset \in D .
\end{array}\right.
$$

Then a representation of a binary relation $\alpha$ of the form $\alpha=\bigcup_{T \in V[\alpha]}\left(Y_{T}^{\alpha} \times T\right)$ is called quasinormal. Note that, if $\alpha=\bigcup_{T \in V[\alpha]}\left(Y_{T}^{\alpha} \times T\right)$ is a quasinormal representation of the binary relation $\alpha$, then $X=\bigcup_{T \in V\left(X^{*}, \alpha\right)} Y_{T}^{\alpha}$ and $Y_{T}^{\alpha} \cap Y_{T^{\prime}}^{\alpha} \neq \emptyset$ for $T, T^{\prime} \in V\left(X^{*}, \alpha\right)$ which $T \neq T^{\prime}$. In [7] they show that, if $\beta$ is regular element of $B_{X}(D)$, then $V[\beta]=V(D, \beta)$ and a complete $X$-semilattice of unions $D$ is an $X I$ - semilattice of unions if $\Lambda\left(D, D_{t}\right) \in D$ for any $t \in \breve{D}$ and $Z=\bigcup_{t \in Z} \Lambda\left(D, D_{t}\right)$ for any nonempty element $Z$ of $D$.

Let $D^{\prime}$ be an arbitrary nonempty subset of the complete $X$-semilattice of unions $D$. A nonempty element $T \in D^{\prime}$ is a nonlimiting element of $D^{\prime}$ if $T \backslash l\left(D^{\prime}, T\right)=T \backslash \cup\left(D^{\prime} \backslash D_{T}^{\prime}\right) \neq \emptyset$. A nonempty element $T \in D^{\prime}$ is limiting element of $D^{\prime}$ if $T \backslash l\left(D^{\prime}, T\right)=\emptyset$.

The family $C(D)$ of pairwise disjoint subsets of the set $\breve{D}=\cup D$ is the characteristic family of sets of $D$ if the following hold

a) $\cap D \in C(D)$

b) $\cup C(D)=\breve{D}$

c) There exists a subset $C_{Z}(D)$ of the set $C(D)$ such that $Z=\cup C_{Z}(D)$ for all $Z \in D$.

A mapping $\theta: D \rightarrow C(D)$ is called characteristic mapping if $Z=(\cap D) \cup$ $\bigcup \theta\left(Z^{\prime}\right)$ for all $Z \in D$.

$Z^{\prime} \in \hat{D}$

The existence and the uniqueness of characteristic family and characteristic mapping is given in Diasemidze [8]. Moreover, it is shown that every $Z \in D$ can be written as $Z=\theta(\breve{Q}) \cup \bigcup \theta(T)$, where $\hat{Q}(Z)=Q \backslash\{T \in Q \mid Z \subseteq T\}$. $T \in \hat{Q}(Z)$

A one-to-one mapping $\varphi$ between two complete $X$ - semilattices of unions $D^{\prime}$ and $D^{\prime \prime}$ is called a complete isomorphism if $\varphi\left(\cup D_{1}\right)=\underset{T^{\prime} \in D_{1}}{\cup} \varphi\left(T^{\prime}\right)$ for each 
nonempty subset $D_{1}$ of the semilattice $D^{\prime}$. Also, let $\alpha \in B_{X}(D)$. A complete isomorphism $\varphi$ between $X I$-semilattice of unions $Q$ and $D$ is called a complete $\alpha$ - isomorphism if $Q=V(D, \alpha)$ and $\varphi(\emptyset)=\emptyset$ for $\emptyset \in V(D, \alpha)$ and $\varphi(T) \alpha=T$ for any $T \in V(D, \alpha)$.

Let $Q$ and $D^{\prime}$ are respectively some $X I$ and $X$ - subsemilattices of the complete $X$ - semilattice of unions $D$. Then

$R_{\varphi}\left(Q, D^{\prime}\right)=\left\{\alpha \in B_{X}(D) \mid \alpha\right.$ regular element, $\varphi$ complete $\alpha$ - isomorphism $\}$

where $\varphi: Q \rightarrow D^{\prime}$ complete isomorphism and $V(D, \alpha)=Q$. Besides, let us denote

$$
R\left(Q, D^{\prime}\right)=\bigcup_{\varphi \in \Phi\left(Q, D^{\prime}\right)} R_{\varphi}\left(Q, D^{\prime}\right) \text { and } R\left(D^{\prime}\right)=\bigcup_{Q^{\prime} \in \Omega(Q)} R\left(Q^{\prime}, D^{\prime}\right) .
$$

where

$\Phi\left(Q, D^{\prime}\right)=\left\{\varphi \mid \varphi: Q \rightarrow D^{\prime}\right.$ is a complete $\alpha$-isomorhism $\left.\exists \alpha \in B_{X}(D)\right\}$,

$\Omega(Q)=$

$\left\{Q^{\prime} \mid Q^{\prime}\right.$ is $X I$-subsemilattices of $D$ which is complete isomorphic to $\left.Q\right\}$.

E. Schröder described the theory of binary relations in detail in the 1890s ([1]). The basic concepts and the properties of the theory were introduced in "Principia mathemetica" Whitehead and Russell([2]). The theory of binary relations has been improved by Riguet ([3] - [4]). Many researcher studied this theory using partial transformations as Vagner did ([5]). Regular elements of semigroup play an importent role in semigroup theory. Therefore Diasamidze generate systmatic rules for understanding structure of a semigroup of binary relations and characterization of regular elements of these semigroup in $([6]-[9])$. In general he studied semigroups but, in particular, he investigates complete semigroups of the binary relations.

In this paper, we take in particular, $Q=\left\{T_{1}, T_{2}, T_{3}, T_{4}, T_{5}, T_{6}, T_{7}, T_{8}\right\}$ subsemilattice of $X$-semilattice of unions $D$ where the elements $T_{i}$ ' s, $i=1,2, \ldots, 8$ are satisfying the following properties, $T_{1} \subset T_{2} \subset T_{3} \subset T_{5} \subset T_{6} \subset T_{8}$, $T_{1} \subset T_{2} \subset T_{3} \subset T_{5} \subset T_{7} \subset T_{8}, T_{1} \subset T_{2} \subset T_{4} \subset T_{5} \subset T_{6} \subset T_{8}, T_{1} \subset$ $T_{2} \subset T_{4} \subset T_{5} \subset T_{7} \subset T_{8}, T_{1} \neq \emptyset, T_{4} \backslash T_{3} \neq \emptyset, T_{3} \backslash T_{4} \neq \emptyset, T_{6} \backslash T_{7} \neq \emptyset, T_{7} \backslash T_{6} \neq \emptyset$, $T_{3} \cup T_{4}=T_{5}, T_{6} \cup T_{7}=T_{8}$. We will investigate the properties of regular elemant $\alpha \in B_{X}(D)$ satisfying $V(D, \alpha)=Q$. Moreover, we will calculate the number of regular elements of $B_{X}(D)$ for a finite set $X$. 
As general, we study the properties and calculate the number of regular elements of $B_{X}(D)$ satisfying $V(D, \alpha)=Q^{\prime}$ where $Q^{\prime}$ is a semilattice isomorph to $Q$. So, we characterize the class for each element of which is isomorphic to $Q$ by means of the characteristic family of sets, the characteristic mapping and the generate set of $D$.

\section{Preliminaries}

Theorem 2.1. [9, Theorem 10] Let $\alpha$ and $\sigma$ be binary relations of the semigroup $B_{X}(D)$ such that $\alpha \circ \sigma \circ \alpha=\alpha$. If $D(\alpha)$ is some generating set of the semilattice $V(D, \alpha) \backslash\{\emptyset\}$ and $\alpha=\bigcup_{T \in V(D, \alpha)}\left(Y_{T}^{\alpha} \times T\right)$ is a quasinormal representation of the relation $\alpha$, then $V(D, \alpha)$ is a complete $X I$ - semilattice of unions. Moreover, there exists a complete $\alpha$-isomorphism $\varphi$ between the semilattice $V(D, \alpha)$ and $D^{\prime}=\{T \sigma \mid T \in V(D, \alpha)\}$, that satisfies the following conditions:

a) $\varphi(T)=T \sigma$ and $\varphi(T) \alpha=T$ for all $T \in V(D, \alpha)$

b) $\bigcup Y_{T^{\prime}}^{\alpha} \supseteq \varphi(T)$ for any $T \in D(\alpha)$, $T^{\prime} \in \ddot{D}(\alpha)_{T}$

c) $Y_{T}^{\alpha} \cap \varphi(T) \neq \emptyset$ for all nonlimiting element $T$ of the set $\ddot{D}(\alpha)_{T}$,

d) If $T$ is a limiting element of the set $\ddot{D}(\alpha)_{T}$, then the equality $\cup B(T)=T$ is always holds for the set $B(T)=\left\{Z \in \ddot{D}(\alpha)_{T} \mid Y_{Z}^{\alpha} \cap \varphi(T) \neq \emptyset\right\}$.

On the other hand, if $\alpha \in B_{X}(D)$ such that $V(D, \alpha)$ is a complete $X I-$ semilattice of unions. If for a complete $\alpha$-isomorphism $\varphi$ from $V(D, \alpha)$ to a subsemilattice $D^{\prime}$ of $D$ satisfies the conditions $\left.b\right)-d$ ) of the theorem, then $\alpha$ is a regular element of $B_{X}(D)$.

Theorem 2.2. [7, Theorem 1.18.2] Let $D_{j}=\left\{T_{1}, \ldots, T_{j}\right\}, X$ be finite set and $\emptyset \neq Y \subseteq X$. If $f$ is a mapping of the set $X$, on the $D_{j}$, for which $f(y)=T_{j}$ for some $y \in Y$, then the numbers of those mappings $f$ of the sets $X$ on the set $D_{j}$ can be calculated by the formula $s=j^{|X \backslash Y|} \cdot\left(j^{|Y|}-(j-1)^{|Y|}\right)$.

Theorem 2.3. [7, Theorem 6.3.5] Let $X$ is a finite set. If $\varphi$ is a fixed element of the set $\Phi\left(D, D^{\prime}\right)$ and $|\Omega(D)|=m_{0}$ and $q$ is a number of all automorphisms of the semilattice $D$ then $\left|R\left(D^{\prime}\right)\right|=m_{0} \cdot q \cdot\left|R_{\varphi}\left(D, D^{\prime}\right)\right|$. 


\section{Results}

Let $X$ be a finite set, $D$ be a complete $X$-semilattice of unions and $Q=$ $\left\{T_{1}, T_{2}, T_{3}, T_{4}, T_{5}, T_{6}, T_{7}, T_{8}\right\}$ be $X$-subsemilattice of unions of $D$ satisfies the following conditions

$$
\begin{array}{ll}
T_{1} \subset T_{2} \subset T_{3} \subset T_{5} \subset T_{6} \subset T_{8}, & T_{1} \subset T_{2} \subset T_{3} \subset T_{5} \subset T_{7} \subset T_{8}, \\
T_{1} \subset T_{2} \subset T_{4} \subset T_{5} \subset T_{6} \subset T_{8}, & T_{1} \subset T_{2} \subset T_{4} \subset T_{5} \subset T_{7} \subset T_{8}, \\
T_{4} \backslash T_{3} \neq \emptyset, T_{3} \backslash T_{4} \neq \emptyset, & T_{6} \backslash T_{7} \neq \emptyset, T_{7} \backslash T_{6} \neq \emptyset, \\
T_{3} \cup T_{4}=T_{5}, T_{6} \cup T_{7}=T_{8} & T_{1} \neq \emptyset .
\end{array}
$$

The diagram of the $Q$ is shown in Figure 3.1. Let $C(Q)=$

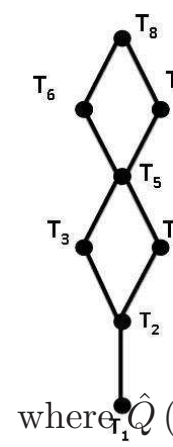
$\left\{P_{1}, P_{2}, P_{3}, P_{4}, P_{5}, P_{6}, P_{7}, P_{8}\right\}$ is characteristic family of sets of $Q$ and $\theta: Q \rightarrow C(Q), \theta\left(T_{i}\right)=P_{i}(i=1,2, \ldots, 8)$ is characteristic mapping.

Then, by using properties of characteristic family and characteristic mapping for each element $T_{i} \in Q$ we can write

$$
T_{i}=\theta(\breve{Q}) \cup \bigcup_{T \in \hat{Q}\left(T_{i}\right)} \theta(T),(i=1,2, \ldots, 8)
$$

Hence,

$$
\begin{aligned}
& T_{8}=P_{8} \cup \bigcup^{T \in \hat{Q}\left(T_{8}\right)} \theta(T)=P_{8} \cup P_{7} \cup P_{6} \cup P_{5} \cup P_{4} \cup P_{3} \cup P_{2} \cup P_{1}, \\
& T_{7}=P_{8} \cup \bigcup^{T \in \hat{Q}\left(T_{7}\right)} \theta(T)=P_{8} \cup P_{6} \cup P_{5} \cup P_{4} \cup P_{3} \cup P_{2} \cup P_{1}, \\
& T_{6}=P_{8} \cup \bigcup_{T \in \hat{Q}\left(T_{6}\right)}^{\theta} \theta(T)=P_{8} \cup P_{7} \cup P_{5} \cup P_{4} \cup P_{3} \cup P_{2} \cup P_{1}, \\
& T_{5}=P_{8} \cup \bigcup^{T \in \hat{Q}\left(T_{5}\right)} \theta(T)=P_{8} \cup P_{4} \cup P_{3} \cup P_{2} \cup P_{1}, \\
& T_{4}=P_{8} \cup \bigcup^{T \in \hat{Q}\left(T_{4}\right)} \theta(T)=P_{8} \cup P_{3} \cup P_{2} \cup P_{1}, \\
& T_{3}=P_{8} \cup \bigcup_{T \in \hat{Q}\left(T_{3}\right)}^{\theta} \theta(T)=P_{8} \cup P_{4} \cup P_{2} \cup P_{1}, \\
& T_{2}=P_{8} \cup \bigcup_{T \in \hat{Q}\left(T_{2}\right)}^{\theta} \theta(T)=P_{8} \cup P_{1}, \\
& T_{1}=P_{8} \cup \bigcup_{T \in \hat{Q}\left(T_{1}\right)}^{\theta} \theta(T)=P_{8} \cup \emptyset=P_{8}
\end{aligned}
$$


are obtained.

Lemma 3.1. $Q$ is $X I-$ semilattice of unions.

Proof. Let us show that the conditions of definition of $X I$ - semilattice of unions hold. First, let determine the greatest lower bounds of the each semilattice $Q_{t}$ in $Q$ for $t \in T_{8}$. Since $T_{8}=P_{8} \cup P_{7} \cup P_{6} \cup P_{5} \cup P_{4} \cup P_{3} \cup P_{2} \cup P_{1}$ and $P_{i}(i=1,2, \ldots, 8)$ are pairwise disjoint sets, by Equation (3.1) and the definition of $Q_{t}$, we get

$$
Q_{t}= \begin{cases}Q & , t \in P_{8} \\ \left\{T_{8}, T_{6}\right\} & , t \in P_{7} \\ \left\{T_{8}, T_{7}\right\} & , t \in P_{6} \\ \left\{T_{8}, T_{7}, T_{6}\right\} & , t \in P_{5} \\ \left\{T_{8}, T_{7}, T_{6}, T_{5}, T_{3}\right\} & , t \in P_{4} \\ \left\{T_{8}, T_{7}, T_{6}, T_{5}, T_{4}\right\} & , t \in P_{3} \\ \left\{T_{8}, T_{7}, T_{6}, T_{5}, T_{4}, T_{3}\right\} & , t \in P_{2} \\ \left\{T_{8}, T_{7}, T_{6}, T_{5}, T_{4}, T_{3}, T_{2}\right\} & , t \in P_{1}\end{cases}
$$

By using Equation (3.2) and the definition of $N\left(Q, Q_{t}\right)$, we get

$$
N\left(Q, Q_{t}\right)= \begin{cases}\left\{T_{1}\right\} & , t \in P_{8} \\ \left\{T_{1}, T_{2}, T_{3}, T_{4}, T_{5}, T_{6}\right\} & , t \in P_{7} \\ \left\{T_{1}, T_{2}, T_{3}, T_{4}, T_{5}, T_{7}\right\} & , t \in P_{6} \\ \left\{T_{1}, T_{2}, T_{3}, T_{4}, T_{5}\right\} & , t \in P_{5} \\ \left\{T_{1}, T_{2}, T_{3}\right\} & , t \in P_{4} \\ \left\{T_{1}, T_{2}, T_{4}\right\} & , t \in P_{3} \\ \left\{T_{1}, T_{2}\right\} & , t \in P_{2} \\ \left\{T_{1}, T_{2}\right\} & , t \in P_{1}\end{cases}
$$

From the Equation (3.3) the greatest lower bounds for each semilattice $Q_{t}$

$$
\cup N\left(Q, Q_{t}\right)=\Lambda\left(Q, Q_{t}\right)= \begin{cases}T_{1} & , t \in P_{8} \\ T_{6} & , t \in P_{7} \\ T_{7} & , t \in P_{6} \\ T_{5} & , t \in P_{5} \\ T_{3} & , t \in P_{4} \\ T_{4} & , t \in P_{3} \\ T_{2} & , t \in P_{2} \\ T_{2} & , t \in P_{1}\end{cases}
$$


are obtained. So, we get $\Lambda\left(D, D_{t}\right) \in D$ for any $t \in T_{8}$. Now Using the Equation (3.4), we have

$$
\begin{aligned}
& t \in T_{1}=P_{8} \Rightarrow T_{1}=\Lambda\left(Q, Q_{t}\right), \\
& t \in T_{2}=P_{8} \cup P_{1} \Rightarrow t \in P_{8} \text { or } t \in P_{1} \Rightarrow \Lambda\left(Q, Q_{t}\right) \in\left\{T_{1}, T_{2}\right\} \\
& \Rightarrow T_{2}=T_{1} \cup T_{2}=\bigcup_{t \in T_{2}} \Lambda\left(Q, Q_{t}\right), \\
& t \in T_{3}=P_{8} \cup P_{4} \cup P_{2} \cup P_{1} \Rightarrow \Rightarrow \Lambda\left(Q, Q_{t}\right) \in\left\{T_{1}, T_{2}, T_{3}\right\} \\
& \Rightarrow T_{3}=T_{1} \cup T_{2} \cup T_{3}=\bigcup_{t \in T_{3}} \Lambda\left(Q, Q_{t}\right), \\
& t \in T_{4}=P_{8} \cup P_{3} \cup P_{2} \cup P_{1} \Rightarrow \Rightarrow \Lambda\left(Q, Q_{t}\right) \in\left\{T_{1}, T_{2}, T_{4}\right\} \\
& \Rightarrow T_{4}=T_{1} \cup T_{2} \cup T_{4}=\bigcup_{t \in T_{4}} \Lambda\left(Q, Q_{t}\right), \\
& t \in T_{5}=P_{8} \cup P_{4} \cup P_{3} \cup P_{2} \cup P_{1} \Rightarrow \Lambda\left(Q, Q_{t}\right)=\left\{T_{1}, T_{2}, T_{3}, T_{4}\right\} \\
& \Rightarrow T_{5}=T_{1} \cup T_{2} \cup T_{3} \cup T_{4}=\bigcup_{t \in T_{5}} \Lambda\left(Q, Q_{t}\right), \\
& t \in T_{6}=P_{8} \cup P_{7} \cup P_{5} \cup \ldots \cup P_{1} \Rightarrow \Lambda\left(Q, Q_{t}\right)=\left\{T_{1}, T_{2}, T_{3}, T_{4}, T_{5}, T_{6}\right\} \\
& \Rightarrow T_{6}=T_{1} \cup \ldots \cup T_{6}=\bigcup_{t \in T_{6}} \Lambda\left(Q, Q_{t}\right), \\
& t \in T_{7}=P_{8} \cup P_{6} \cup P_{5} \cup \ldots \cup P_{1} \Rightarrow \Lambda\left(Q, Q_{t}\right)=\left\{T_{1}, T_{2}, T_{3}, T_{4}, T_{5}, T_{7}\right\} \\
& \Rightarrow T_{7}=T_{1} \cup \ldots \cup T_{5} \cup T_{7}=\bigcup_{t \in T_{7}} \Lambda\left(Q, Q_{t}\right), \\
& t \in T_{8}=T_{7} \cup T_{6} \Rightarrow \Lambda\left(Q, Q_{t}\right)=\left\{T_{1}, T_{2}, T_{3}, T_{4}, T_{5}, T_{6}, T_{7}\right\} \\
& \Rightarrow T_{8}=T_{6} \cup T_{7}=\bigcup_{t \in T_{8}} \Lambda\left(Q, Q_{t}\right) \text {. }
\end{aligned}
$$

Then $Q$ is a $X I-$ semilattice of unions.

Lemma 3.2. Following equalities are true for $Q$ where $P_{i}$ 's are pairwise disjoint sets and union of these sets equals $Q$.

$$
\begin{aligned}
& P_{1}=T_{2} \backslash T_{1}, \quad P_{2}=\left(T_{4} \cap T_{3}\right) \backslash T_{2}, \quad P_{3}=T_{4} \backslash T_{3}, \quad P_{4}=T_{3} \backslash T_{4}, \\
& P_{5}=\left(T_{7} \cap T_{6}\right) \backslash T_{5}, \quad P_{6}=T_{7} \backslash T_{6}, \quad P_{7}=T_{6} \backslash T_{7}, \quad P_{8}=T_{1} \text {. }
\end{aligned}
$$

Proof. Considering the (3.1), it is easy to see that equalities are true.

Lemma 3.3. Let $G=\left\{T_{1}, T_{2}, T_{3}, T_{4}, T_{5}, T_{6}, T_{7}\right\}$ be a generating set of $Q$. Then the elements $T_{1}, T_{2}, T_{3}, T_{4}, T_{6}, T_{7}$ are nonlimiting elements of the set $\ddot{G}_{T_{1}}$, $\ddot{G}_{T_{2}}, \ddot{G}_{T_{3}}, \ddot{G}_{T_{4}}, \ddot{G}_{T_{6}}, \ddot{G}_{T_{7}}$ respectively and $T_{5}$ is limiting eleman of the set $\ddot{G}_{T_{5}}$. 
Proof. Definition of $\ddot{D}_{T}^{\prime}$, following equations

$$
\begin{aligned}
& \ddot{G}_{T_{1}}=\left\{T_{1}\right\}, \\
& \ddot{G}_{T_{2}}=\left\{T_{1}, T_{2}\right\}, \\
& \ddot{G}_{T_{3}}=\left\{T_{1}, T_{2}, T_{3}\right\}, \\
& \ddot{G}_{T_{4}}=\left\{T_{1}, T_{2}, T_{4}\right\}, \\
& \ddot{G}_{T_{5}}=\left\{T_{1}, T_{2}, T_{3}, T_{4}, T_{5}\right\}, \\
& \ddot{G}_{T_{6}}=\left\{T_{1}, T_{2}, T_{3}, T_{4}, T_{5}, T_{6}\right\}, \\
& \ddot{G}_{T_{7}}=\left\{T_{1}, T_{2}, T_{3}, T_{4}, T_{5}, T_{7}\right\} .
\end{aligned}
$$

are obtained. Now we get the sets $l\left(\ddot{G}_{T_{i}}, T_{i}\right), i \in\{1,2, \ldots, 7\}$,

$$
\begin{aligned}
& l\left(\ddot{G}_{T_{1}}, T_{1}\right)=\cup\left(\ddot{G}_{T_{1}} \backslash\left\{T_{1}\right\}\right)=\emptyset, \\
& l\left(\ddot{G}_{T_{2}}, T_{2}\right)=\cup\left(\ddot{G}_{T_{2}} \backslash\left\{T_{2}\right\}\right)=T_{1}, \\
& l\left(\ddot{G}_{T_{3}}, T_{3}\right)=\cup\left(\ddot{G}_{T_{3}} \backslash\left\{T_{3}\right\}\right)=T_{2}, \\
& l\left(\ddot{G}_{T_{4}}, T_{4}\right)=\cup\left(\ddot{G}_{T_{4}} \backslash\left\{T_{4}\right\}\right)=T_{2}, \\
& l\left(\ddot{G}_{T_{5}}, T_{5}\right)=\cup\left(\ddot{G}_{T_{5}} \backslash\left\{T_{5}\right\}\right)=T_{5}, \\
& l\left(\ddot{G}_{T_{6}}, T_{6}\right)=\cup\left(\ddot{G}_{T_{6}} \backslash\left\{T_{6}\right\}\right)=T_{5}, \\
& l\left(\ddot{G}_{T_{7}}, T_{7}\right)=\cup\left(\ddot{G}_{T_{7}} \backslash\left\{T_{7}\right\}\right)=T_{5} .
\end{aligned}
$$

Then we find nonlimiting and limiting elements of $\ddot{G}_{T_{i}}, i \in\{1,2, \ldots, 7\}$.

$$
\begin{aligned}
& T_{1} \backslash l\left(\ddot{G}_{T_{1}}, T_{1}\right)=T_{1} \backslash \emptyset \quad=T_{1} \neq \emptyset, \quad T_{1} \text { nonlimiting element } \\
& T_{2} \backslash l\left(\ddot{G}_{T_{2}}, T_{2}\right)=T_{2} \backslash T_{1} \neq \emptyset \quad T_{2} \text { nonlimiting element } \\
& T_{3} \backslash l\left(\ddot{G}_{T_{3}}, T_{3}\right)=T_{3} \backslash T_{2} \neq \emptyset \quad T_{3} \text { nonlimiting element } \\
& T_{4} \backslash l\left(\ddot{G}_{T_{4}}, T_{4}\right)=T_{4} \backslash T_{2} \neq \emptyset \quad T_{4} \text { nonlimiting element } \\
& T_{5} \backslash l\left(\ddot{G}_{T_{5}}, T_{5}\right)=T_{5} \backslash T_{5}=\emptyset \quad T_{5} \text { limiting element } \\
& T_{6} \backslash l\left(\ddot{G}_{T_{6}}, T_{6}\right)=T_{6} \backslash T_{5} \neq \emptyset \quad T_{6} \text { nonlimiting element } \\
& T_{7} \backslash l\left(\ddot{G}_{T_{7}}, T_{7}\right)=T_{7} \backslash T_{5} \neq \emptyset \quad T_{7} \text { nonlimiting element }
\end{aligned}
$$

Therefore, the elements $T_{1}, T_{2}, T_{3}, T_{4}, T_{6}, T_{7}$ are nonlimiting elements of the sets $\ddot{G}_{T_{1}}, \ddot{G}_{T_{2}}, \ddot{G}_{T_{3}}, \ddot{G}_{T_{4}}, \ddot{G}_{T_{6}}, \ddot{G}_{T_{7}}$, respectively and $T_{5}$ is limiting eleman of the set $\ddot{G}_{T_{5}}$.

Now, we determine properties of a reguler element $\alpha$ of $B_{X}(Q)$ where $V(D, \alpha)=Q$ and $\alpha=\bigcup_{i=1}^{8}\left(Y_{i}^{\alpha} \times T_{i}\right)$.

Theorem 3.4. Let $\alpha \in B_{X}(Q)$ be a quasinormal representation of the form $\alpha=\bigcup_{i=1}^{8}\left(Y_{i}^{\alpha} \times T_{i}\right)$ such that $V(D, \alpha)=Q . \alpha \in B_{X}(D)$ is a regular iff for 
some complete $\alpha$-isomorphism $\varphi: Q \rightarrow D^{\prime} \subseteq D$, the following conditions are satisfied:

$$
\begin{aligned}
& Y_{1}^{\alpha} \supseteq \varphi\left(T_{1}\right), \\
& Y_{1}^{\alpha} \cup Y_{2}^{\alpha} \supseteq \varphi\left(T_{2}\right), \\
& Y_{1}^{\alpha} \cup Y_{2}^{\alpha} \cup Y_{3}^{\alpha} \supseteq \varphi\left(T_{3}\right), \\
& Y_{1}^{\alpha} \cup Y_{2}^{\alpha} \cup Y_{4}^{\alpha} \supseteq \varphi\left(T_{4}\right), \\
& Y_{1}^{\alpha} \cup Y_{2}^{\alpha} \cup Y_{3}^{\alpha} \cup Y_{4}^{\alpha} \cup Y_{5}^{\alpha} \cup Y_{6}^{\alpha} \supseteq \varphi\left(T_{6}\right), \\
& Y_{1}^{\alpha} \cup Y_{2}^{\alpha} \cup Y_{3}^{\alpha} \cup Y_{4}^{\alpha} \cup Y_{5}^{\alpha} \cup Y_{7}^{\alpha} \supseteq \varphi\left(T_{7}\right), \\
& Y_{2}^{\alpha} \cap \varphi\left(T_{2}\right) \neq \emptyset, Y_{3}^{\alpha} \cap \varphi\left(T_{3}\right) \neq \emptyset, \\
& Y_{4}^{\alpha} \cap \varphi\left(T_{4}\right) \neq \emptyset, Y_{6}^{\alpha} \cap \varphi\left(T_{6}\right) \neq \emptyset, \\
& Y_{7}^{\alpha} \cap \varphi\left(T_{7}\right) \neq \emptyset .
\end{aligned}
$$

Proof. Let $G=\left\{T_{1}, T_{2}, T_{3}, T_{4}, T_{5}, T_{6}, T_{7}\right\}$ be a generating set of $Q$.

$\Rightarrow$ : Since $\alpha \in B_{X}(D)$ is regular and $V(D, \alpha)=Q X I$-semilattice of unions, by Theorem 2.1, there exits a complete isomorphism $\varphi: Q \rightarrow D^{\prime}$. By Theorem $2.1(a)$, satisfying $\varphi(T) \alpha=T$ for all $T \in V(D, \alpha)$. So, $\varphi$ is complete $\alpha-$ isomorphism. Applying the Theorem $2.1(b)$ we have

$$
\begin{aligned}
& Y_{1}^{\alpha} \supseteq \varphi\left(T_{1}\right) \\
& Y_{1}^{\alpha} \cup Y_{2}^{\alpha} \supseteq \varphi\left(T_{2}\right) \\
& Y_{1}^{\alpha} \cup Y_{2}^{\alpha} \cup Y_{3}^{\alpha} \supseteq \varphi\left(T_{3}\right) \\
& Y_{1}^{\alpha} \cup Y_{2}^{\alpha} \cup Y_{4}^{\alpha} \supseteq \varphi\left(T_{4}\right) \\
& Y_{1}^{\alpha} \cup Y_{2}^{\alpha} \cup Y_{3}^{\alpha} \cup Y_{4}^{\alpha} \cup Y_{5}^{\alpha} \supseteq \varphi\left(T_{5}\right) \\
& Y_{1}^{\alpha} \cup Y_{2}^{\alpha} \cup Y_{3}^{\alpha} \cup Y_{4}^{\alpha} \cup Y_{5}^{\alpha} \cup Y_{6}^{\alpha} \supseteq \varphi\left(T_{6}\right), \\
& Y_{1}^{\alpha} \cup Y_{2}^{\alpha} \cup Y_{3}^{\alpha} \cup Y_{4}^{\alpha} \cup Y_{5}^{\alpha} \cup Y_{7}^{\alpha} \supseteq \varphi\left(T_{7}\right)
\end{aligned}
$$

Moreover, considering that the elements $T_{1}, T_{2}, T_{3}, T_{4}, T_{6}, T_{7}$ are nonlimiting and using the Theorem $2.1(c)$, following properties

$$
\begin{aligned}
& Y_{1}^{\alpha} \cap \varphi\left(T_{1}\right) \neq \emptyset, Y_{2}^{\alpha} \cap \varphi\left(T_{2}\right) \neq \emptyset, \\
& Y_{3}^{\alpha} \cap \varphi\left(T_{3}\right) \neq \emptyset, Y_{4}^{\alpha} \cap \varphi\left(T_{4}\right) \neq \emptyset, \\
& Y_{6}^{\alpha} \cap \varphi\left(T_{6}\right) \neq \emptyset, Y_{7}^{\alpha} \cap \varphi\left(T_{7}\right) \neq \emptyset .
\end{aligned}
$$

are obtained. From $Y_{1}^{\alpha} \supseteq \varphi\left(T_{1}\right), Y_{1}^{\alpha} \cap \varphi\left(T_{1}\right) \neq \emptyset$ always ensured. Also by using $Y_{1}^{\alpha} \cup Y_{2}^{\alpha} \cup Y_{3}^{\alpha} \supseteq \varphi\left(T_{3}\right)$ and $Y_{1}^{\alpha} \cup Y_{2}^{\alpha} \cup Y_{4}^{\alpha} \supseteq \varphi\left(T_{4}\right)$, we get

$$
\begin{aligned}
Y_{1}^{\alpha} \cup Y_{2}^{\alpha} \cup Y_{3}^{\alpha} \cup Y_{4}^{\alpha} \cup Y_{5}^{\alpha} & =\left(Y_{1}^{\alpha} \cup Y_{2}^{\alpha} \cup Y_{3}^{\alpha}\right) \cup\left(Y_{1}^{\alpha} \cup Y_{2}^{\alpha} \cup Y_{4}^{\alpha}\right) \\
& \supseteq \varphi\left(T_{3}\right) \cup \varphi\left(T_{4}\right) \cup Y_{5}^{\alpha} \\
& =\varphi\left(T_{5}\right) \cup Y_{5}^{\alpha} \\
& \supseteq \varphi\left(T_{5}\right)
\end{aligned}
$$

Thus there is no need the condition $Y_{1}^{\alpha} \cup Y_{2}^{\alpha} \cup Y_{3}^{\alpha} \cup Y_{4}^{\alpha} \cup Y_{5}^{\alpha} \supseteq \varphi\left(T_{5}\right)$. Therefore there exist an $\alpha$-isomorphism $\varphi$ which holds given conditions. 
$\Leftarrow$ : Since $V(D, \alpha)=Q, V(D, \alpha)$ is $X I$-semilattice of unions. Let $\varphi$ : $Q \rightarrow D^{\prime} \subseteq D$ be complete $\alpha$-isomorphism which holds given conditions. So, considering Equation (3.6), satisfying Theorem $2.1(a)-(c)$. Remembering that $T_{5}$ is a limiting element of the set $\ddot{G}_{T_{5}}$, we constitute the set $B\left(T_{5}\right)=$ $\left\{Z \in \ddot{G}_{T_{5}} \mid Y_{Z}^{\alpha} \cap \varphi\left(T_{5}\right) \neq \emptyset\right\}$. If $Y_{4}^{\alpha} \cap \varphi\left(T_{5}\right)=\emptyset$ we have

$$
\begin{aligned}
Y_{1}^{\alpha} \cup Y_{2}^{\alpha} \cup Y_{3}^{\alpha} \cup Y_{4}^{\alpha} & =\left(Y_{1}^{\alpha} \cup Y_{2}^{\alpha} \cup Y_{3}^{\alpha}\right) \cup\left(Y_{1}^{\alpha} \cup Y_{2}^{\alpha} \cup Y_{4}^{\alpha}\right) \\
& \supseteq \varphi\left(T_{3}\right) \cup \varphi\left(T_{4}\right)=\varphi\left(T_{5}\right)
\end{aligned}
$$

So we get $Y_{1}^{\alpha} \cup Y_{2}^{\alpha} \cup Y_{3}^{\alpha} \supseteq \varphi\left(T_{5}\right) \supseteq \varphi\left(T_{4}\right)$ which is a contradiction with $Y_{4}^{\alpha} \cap \varphi\left(T_{4}\right) \neq \emptyset$. Therefore $T_{4} \in B\left(T_{5}\right)$. If $Y_{3}^{\alpha} \cap \varphi\left(T_{5}\right)=\emptyset$ we have

$$
\begin{aligned}
Y_{1}^{\alpha} \cup Y_{2}^{\alpha} \cup Y_{3}^{\alpha} \cup Y_{4}^{\alpha} & =\left(Y_{1}^{\alpha} \cup Y_{2}^{\alpha} \cup Y_{3}^{\alpha}\right) \cup\left(Y_{1}^{\alpha} \cup Y_{2}^{\alpha} \cup Y_{4}^{\alpha}\right) \\
& \supseteq \varphi\left(T_{3}\right) \cup \varphi\left(T_{4}\right)=\varphi\left(T_{5}\right)
\end{aligned}
$$

So we get $Y_{1}^{\alpha} \cup Y_{2}^{\alpha} \cup Y_{4}^{\alpha} \supseteq \varphi\left(T_{5}\right) \supseteq \varphi\left(T_{3}\right)$ which is a contradiction with $Y_{3}^{\alpha} \cap \varphi\left(T_{3}\right) \neq \emptyset$. Therefore $T_{3} \in B\left(T_{5}\right)$. We have $\cup B\left(T_{5}\right)=T_{3} \cup T_{4}=T_{5}$. By Theorem 2.1, we conclude that $\alpha$ is the regular element of the $B_{X}(D)$.

Now we calculate the number of regular elements $\alpha$, satisfying the hyphothesis of Theorem 3.4. Let $\alpha \in B_{X}(D)$ be a regular element which is quasinormal representation of the form $\alpha=\bigcup_{i=1}^{8}\left(Y_{i}^{\alpha} \times T_{i}\right)$ and $V(D, \alpha)=Q$. Then there exist a complete $\alpha$ - isomorphism $\varphi: Q \rightarrow D^{\prime}=\left\{\varphi\left(T_{1}\right), \varphi\left(T_{2}\right), \ldots, \varphi\left(T_{8}\right)\right\}$ satisfying the hyphothesis of Theorem 3.4. So, $\alpha \in R_{\varphi}\left(Q, D^{\prime}\right)$. We will denote $\varphi\left(T_{i}\right)=$ $\bar{T}_{i}, i=1,2, \ldots 8$. Diagram of the $D^{\prime}=\left\{\bar{T}_{1}, \bar{T}_{2}, \bar{T}_{3}, \bar{T}_{4}, \bar{T}_{5}, \bar{T}_{6}, \bar{T}_{7}, \bar{T}_{8}\right\}$ is shown in Figure 3.2. Then the Equation (3.6) reduced to below equation.

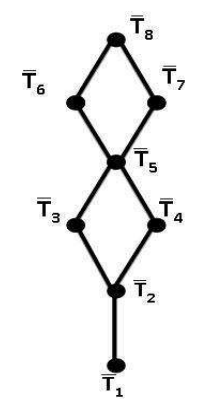

Figure 3.2

$$
\begin{aligned}
& Y_{1}^{\alpha} \supseteq \bar{T}_{1} \\
& Y_{1}^{\alpha} \cup Y_{2}^{\alpha} \supseteq \bar{T}_{2} \\
& Y_{1}^{\alpha} \cup Y_{2}^{\alpha} \cup Y_{3}^{\alpha} \supseteq \bar{T}_{3} \\
& Y_{1}^{\alpha} \cup Y_{2}^{\alpha} \cup Y_{4}^{\alpha} \supseteq \bar{T}_{4} \\
& Y_{1}^{\alpha} \cup Y_{2}^{\alpha} \cup Y_{3}^{\alpha} \cup Y_{4}^{\alpha} \cup Y_{5}^{\alpha} \cup Y_{6}^{\alpha} \supseteq \bar{T}_{6}, \\
& Y_{1}^{\alpha} \cup Y_{2}^{\alpha} \cup Y_{3}^{\alpha} \cup Y_{4}^{\alpha} \cup Y_{5}^{\alpha} \cup Y_{7}^{\alpha} \supseteq \bar{T}_{7} \\
& Y_{2}^{\alpha} \cap \varphi\left(T_{2}\right) \neq \emptyset, Y_{3}^{\alpha} \cap \varphi\left(T_{3}\right) \neq \emptyset, \\
& Y_{4}^{\alpha} \cap \varphi\left(T_{4}\right) \neq \emptyset, Y_{6}^{\alpha} \cap \varphi\left(T_{6}\right) \neq \emptyset, \\
& Y_{7}^{\alpha} \cap \varphi\left(T_{7}\right) \neq \emptyset .
\end{aligned}
$$

On the other hand, the image of the sets in Lemma 3.2 under

$$
\bar{T}_{1},\left(\bar{T}_{3} \cap \bar{T}_{4}\right) \backslash \bar{T}_{1}, \bar{T}_{4} \backslash \bar{T}_{3}, \bar{T}_{3} \backslash \bar{T}_{4},\left(\bar{T}_{7} \cap \bar{T}_{6}\right) \backslash \bar{T}_{5}, \bar{T}_{7} \backslash \bar{T}_{6}, \bar{T}_{6} \backslash \bar{T}_{7}, X \backslash \bar{T}_{8}
$$


are also pairwise disjoint sets and union of these sets equals $X$.

Lemma 3.5. For every $\alpha \in R_{\varphi}\left(Q, D^{\prime}\right)$, there exists an ordered system of disjoint mappings which is defined $\left\{\bar{T}_{1},\left(\bar{T}_{3} \cap \bar{T}_{4}\right) \backslash \bar{T}_{1}, \bar{T}_{4} \backslash \bar{T}_{3}, \bar{T}_{3} \backslash \bar{T}_{4},\left(\bar{T}_{7} \cap\right.\right.$ $\left.\left.\bar{T}_{6}\right) \backslash \bar{T}_{5}, \bar{T}_{7} \backslash \bar{T}_{6}, \bar{T}_{6} \backslash \bar{T}_{7}, X \backslash \bar{T}_{8}\right\}$. Also, ordered systems are different which correspond to different binary relations.

Proof. Let $f_{\alpha}: X \rightarrow D$ be a mapping satisfying the condition $f_{\alpha}(t)=t \alpha$ for all $t \in X$. We consider the restrictions of the mapping $f_{\alpha}$ as $f_{1 \alpha}, f_{2 \alpha}$, $f_{3 \alpha}, f_{4 \alpha}, f_{5 \alpha}, f_{6 \alpha}, f_{7 \alpha}, f_{8 \alpha}$ on the sets $\bar{T}_{1},\left(\bar{T}_{3} \cap \bar{T}_{4}\right) \backslash \bar{T}_{1}, \bar{T}_{4} \backslash \bar{T}_{3}, \bar{T}_{3} \backslash \bar{T}_{4},\left(\bar{T}_{7} \cap\right.$ $\left.\bar{T}_{6}\right) \backslash \bar{T}_{5}, \bar{T}_{7} \backslash \bar{T}_{6}, \bar{T}_{6} \backslash \bar{T}_{7}, X \backslash \bar{T}_{8}$, respectively.

Now, considering the definition of the sets $Y_{i}^{\alpha}, i=1,2, \ldots, 8$, together with the Equation (3.9) we have

$$
\begin{aligned}
t \in \bar{T}_{1} \Rightarrow t \in Y_{1}^{\alpha} & \Rightarrow t \alpha=T_{1} \Rightarrow f_{1 \alpha}(t)=T_{1}, \forall t \in \bar{T}_{1} . \\
t \in\left(\bar{T}_{3} \cap \bar{T}_{4}\right) \backslash \bar{T}_{1} & \Rightarrow t \in\left(\bar{T}_{3} \cap \bar{T}_{4}\right) \subseteq Y_{1}^{\alpha} \cup Y_{2}^{\alpha} \\
& \Rightarrow t \alpha \in\left\{T_{1}, T_{2}\right\} \\
& \Rightarrow f_{2 \alpha}(t) \in\left\{T_{1}, T_{2}\right\}, \forall t \in\left(\bar{T}_{3} \cap \bar{T}_{4}\right) \backslash \bar{T}_{1} .
\end{aligned}
$$

Since $Y_{2}^{\alpha} \cap \bar{T}_{2} \neq \emptyset$, there is an element $t_{2} \in Y_{2}^{\alpha} \cap \bar{T}_{2}$. Then $t_{2} \alpha=T_{2}$ and $t_{2} \in \bar{T}_{2}$. If $t_{2} \in \bar{T}_{1}$ then $t_{2} \in \bar{T}_{1} \subseteq Y_{1}^{\alpha}$. Therefore, $t_{2} \alpha=T_{1}$ which is in contradiction with the equality $t_{2} \alpha=T_{2}$. So $f_{2 \alpha}\left(t_{2}\right)=T_{2}$ for some $t_{2} \in \bar{T}_{2} \backslash \bar{T}_{1}$.

$$
\begin{aligned}
t \in \bar{T}_{4} \backslash \bar{T}_{3} & \Rightarrow t \in \bar{T}_{4} \backslash \bar{T}_{3} \subseteq \bar{T}_{4} \subseteq Y_{1}^{\alpha} \cup Y_{2}^{\alpha} \cup Y_{4}^{\alpha} \\
& \Rightarrow t \alpha \in\left\{T_{1}, T_{2}, T_{4}\right\} \\
& \Rightarrow f_{3 \alpha}(t) \in\left\{T_{1}, T_{2}, T_{4}\right\}, \forall t \in \bar{T}_{4} \backslash \bar{T}_{3}
\end{aligned}
$$

$Y_{4}^{\alpha} \cap \bar{T}_{4} \neq \emptyset$ so there is an element $t_{4} \in Y_{4}^{\alpha} \cap \bar{T}_{4}$. Then $t_{4} \alpha=T_{4}$ and $t_{4} \in \bar{T}_{4}$. If $t_{4} \in \bar{T}_{3}$ then $t_{4} \in \bar{T}_{3} \subseteq Y_{1}^{\alpha} \cup Y_{2}^{\alpha} \cup Y_{3}^{\alpha}$. Thus $t_{4} \alpha \in\left\{T_{1}, T_{2}, T_{3}\right\}$ which is in contradiction with the equality $t_{4} \alpha=T_{4}$. So there is an element $t_{4} \in \bar{T}_{4} \backslash \bar{T}_{3}$ with $f_{3 \alpha}\left(t_{4}\right)=T_{4}$.

$$
\begin{aligned}
t \in \bar{T}_{3} \backslash \bar{T}_{4} & \Rightarrow t \in \bar{T}_{3} \backslash \bar{T}_{4} \subseteq \bar{T}_{3} \subseteq Y_{1}^{\alpha} \cup Y_{2}^{\alpha} \cup Y_{3}^{\alpha} \\
& \Rightarrow t \alpha \in\left\{T_{1}, T_{2}, T_{3}\right\} \\
& \Rightarrow f_{4 \alpha}(t) \in\left\{T_{1}, T_{2}, T_{3}\right\}, \forall t \in \bar{T}_{3} \backslash \bar{T}_{4}
\end{aligned}
$$

Since $Y_{3}^{\alpha} \cap \bar{T}_{3} \neq \emptyset$, there is an element $t_{3}$ with $t_{3} \alpha=T_{3}$ and $t_{3} \in \bar{T}_{3}$. If $t_{3} \in \bar{T}_{4}$ then $t_{3} \in \bar{T}_{4} \subseteq Y_{1}^{\alpha} \cup Y_{2}^{\alpha} \cup Y_{4}^{\alpha}$. Therefore, $t_{3} \alpha \in\left\{T_{1}, T_{2}, T_{4}\right\}$ which contradicts to the equality $t_{3} \alpha=T_{3}$. So there is an element $t_{3} \in \bar{T}_{3} \backslash \bar{T}_{4}$ with $f_{4 \alpha}\left(t_{3}\right)=T_{3}$.

$$
\begin{aligned}
t \in\left(\bar{T}_{7} \cap \bar{T}_{6}\right) \backslash \bar{T}_{5} & \Rightarrow t \in\left(\bar{T}_{7} \cap \bar{T}_{6}\right) \backslash \bar{T}_{5} \subseteq \bar{T}_{7} \cap \bar{T}_{6} \subseteq Y_{1}^{\alpha} \cup Y_{2}^{\alpha} \cup Y_{3}^{\alpha} \cup Y_{4}^{\alpha} \cup Y_{5}^{\alpha} \\
& \Rightarrow t \alpha \in\left\{T_{1}, T_{2}, T_{3}, T_{4}, T_{5}\right\} \\
& \Rightarrow f_{5 \alpha}(t) \in\left\{T_{1}, T_{2}, T_{3}, T_{4}, T_{5}\right\}, \forall t \in\left(\bar{T}_{7} \cap \bar{T}_{6}\right) \backslash \bar{T}_{5} .
\end{aligned}
$$




$$
\begin{aligned}
t \in \bar{T}_{7} \backslash \bar{T}_{6} & \Rightarrow t \in \bar{T}_{7} \backslash \bar{T}_{6} \subseteq \bar{T}_{7} \subseteq Y_{1}^{\alpha} \cup Y_{2}^{\alpha} \cup Y_{3}^{\alpha} \cup Y_{4}^{\alpha} \cup Y_{5}^{\alpha} \cup Y_{7}^{\alpha} \\
& \Rightarrow t \alpha \in\left\{T_{1}, T_{2}, T_{3}, T_{4}, T_{5}, T_{7}\right\} \\
& \Rightarrow f_{6 \alpha}(t) \in\left\{T_{1}, T_{2}, T_{3}, T_{4}, T_{5}, T_{7}\right\}, \quad \forall t \in \bar{T}_{7} \backslash \bar{T}_{6} .
\end{aligned}
$$

Also, there is an element $t_{7} \in Y_{7}^{\alpha} \cap \bar{T}_{7}$ since $Y_{7}^{\alpha} \cap \bar{T}_{7} \neq \emptyset$. Then $t_{7} \alpha=T_{7}$ and $t_{7} \in \bar{T}_{7}$. If $t_{7} \in \bar{T}_{6}$ then $t_{7} \in \bar{T}_{6} \subseteq Y_{1}^{\alpha} \cup Y_{2}^{\alpha} \cup Y_{3}^{\alpha} \cup Y_{4}^{\alpha} \cup Y_{5}^{\alpha} \cup Y_{6}^{\alpha}$. So $t_{7} \alpha \in$ $\left\{T_{1}, T_{2}, T_{3}, T_{4}, T_{5}, T_{6}\right\}$. However this contradicts to $t_{7} \alpha=T_{7}$. So $f_{6 \alpha}\left(t_{7}\right)=T_{7}$ for some $t_{7} \in \bar{T}_{7} \backslash \bar{T}_{6}$.

$$
\begin{aligned}
t \in \bar{T}_{6} \backslash \bar{T}_{7} & \Rightarrow t \in \bar{T}_{6} \backslash \bar{T}_{7} \subseteq \bar{T}_{6} \subseteq Y_{1}^{\alpha} \cup Y_{2}^{\alpha} \cup Y_{3}^{\alpha} \cup Y_{4}^{\alpha} \cup Y_{5}^{\alpha} \cup Y_{6}^{\alpha} \\
& \Rightarrow t \alpha \in\left\{T_{1}, T_{2}, T_{3}, T_{4}, T_{5}, T_{6}\right\} \\
& \Rightarrow f_{7 \alpha}(t) \in\left\{T_{1}, T_{2}, T_{3}, T_{4}, T_{5}, T_{6}\right\}, \forall t \in \bar{T}_{6} \backslash \bar{T}_{7} .
\end{aligned}
$$

Smilarly there is an element $t_{6}$ with $t_{6} \alpha=T_{6}$ and $t_{6} \in \bar{T}_{6}$ since $Y_{6}^{\alpha} \cap \bar{T}_{6} \neq$ $\emptyset$. If $t_{6} \in \bar{T}_{7}$ then $t_{6} \in \bar{T}_{7} \subseteq Y_{1}^{\alpha} \cup Y_{2}^{\alpha} \cup Y_{3}^{\alpha} \cup Y_{4}^{\alpha} \cup Y_{5}^{\alpha} \cup Y_{7}^{\alpha}$. Therefore, $t_{6} \alpha \in$ $\left\{T_{1}, T_{2}, T_{3}, T_{4}, T_{5}, T_{7}\right\}$ which is in contradiction with the equality $t_{6} \alpha=T_{6}$. So $f_{7 \alpha}\left(t_{6}\right)=T_{6}$ for some $t_{6} \in \bar{T}_{6} \backslash \bar{T}_{7}$.

$$
t \in X \backslash \bar{T}_{8} \Rightarrow t \in X \backslash \bar{T}_{8} \subseteq X=\bigcup_{i=1}^{8} Y_{i}^{\alpha} \Rightarrow t \alpha \in Q \Rightarrow f_{8 \alpha}(t) \in Q, \forall t \in X \backslash \bar{T}_{8}
$$

Therefore, for every binary relation $\alpha \in R_{\varphi}\left(Q, D^{\prime}\right)$ there exists an ordered system $\left(f_{1 \alpha}, f_{2 \alpha}, f_{3 \alpha}, f_{4 \alpha}, f_{5 \alpha}, f_{6 \alpha}, f_{7 \alpha}, f_{8 \alpha}\right)$.

On the other hand, suppose that for $\alpha, \beta \in R_{\varphi}\left(Q, D^{\prime}\right)$ which $\alpha \neq \beta$, be obtained $f_{\alpha}=\left(f_{1 \alpha}, f_{2 \alpha}, f_{3 \alpha}, f_{4 \alpha}, f_{5 \alpha}, f_{6 \alpha}, f_{7 \alpha}, f_{8 \alpha}\right)$ and $f_{\beta}=\left(f_{1 \beta}, f_{2 \beta}, f_{3 \beta}, f_{4 \beta}\right.$, $\left.f_{5 \beta}, f_{6 \beta}, f_{7 \beta}, f_{8 \beta}\right)$. If $f_{\alpha}=f_{\beta}$, we get

$$
f_{\alpha}=f_{\beta} \Rightarrow f_{\alpha}(t)=f_{\beta}(t), \forall t \in X \Rightarrow t \alpha=t \beta, \forall t \in X \Rightarrow \alpha=\beta
$$

which contradicts to $\alpha \neq \beta$. Therefore different binary relations's ordered systems are different.

Lemma 3.6. Let $f=\left(f_{1}, f_{2}, f_{3}, f_{4}, f_{5}, f_{6}, f_{7}, f_{8}\right)$ be ordered system from 
$X$ in the semilattice $D$ such that

$f_{1}: \bar{T}_{1} \rightarrow\left\{T_{1}\right\}, f_{1}(t)=T_{1}$,

$f_{2}:\left(\bar{T}_{3} \cap \bar{T}_{4}\right) \backslash \bar{T}_{1} \rightarrow\left\{T_{1}, T_{2}\right\}, f_{2}(t) \in\left\{T_{1}, T_{2}\right\}$ and $f_{2}\left(t_{2}\right)=T_{2} \exists t_{2} \in \bar{T}_{2} \backslash \bar{T}_{1}$,

$f_{3}: \bar{T}_{4} \backslash \bar{T}_{3} \rightarrow\left\{T_{1}, T_{2}, T_{4}\right\}, f_{3}(t) \in\left\{T_{1}, T_{2}, T_{4}\right\}$ and $f_{3}\left(t_{4}\right)=T_{4} \exists t_{4} \in \bar{T}_{4} \backslash \bar{T}_{3}$,

$f_{4}: \bar{T}_{3} \backslash \bar{T}_{4} \rightarrow\left\{T_{1}, T_{2}, T_{3}\right\}, f_{4}(t) \in\left\{T_{1}, T_{2}, T_{3}\right\}$ and $f_{4}\left(t_{3}\right)=T_{3} \exists t_{3} \in \bar{T}_{3} \backslash \bar{T}_{4}$,

$f_{5}:\left(\bar{T}_{7} \cap \bar{T}_{6}\right) \backslash \bar{T}_{5} \rightarrow\left\{T_{1}, T_{2}, T_{3}, T_{4}, T_{5}\right\}, f_{5}(t) \in\left\{T_{1}, T_{2}, T_{3}, T_{4}, T_{5}\right\}$,

$f_{6}: \bar{T}_{7} \backslash \bar{T}_{6} \rightarrow\left\{T_{1}, T_{2}, T_{3}, T_{4}, T_{5}, T_{7}\right\}, f_{6}(t) \in\left\{T_{1}, T_{2}, T_{3}, T_{4}, T_{5}, T_{7}\right\}$

and $f_{6}\left(t_{7}\right)=T_{7} \exists t_{7} \in \bar{T}_{7} \backslash \bar{T}_{6}$,

$f_{7}: \bar{T}_{6} \backslash \bar{T}_{7} \rightarrow\left\{T_{1}, T_{2}, T_{3}, T_{4}, T_{5}, T_{6}\right\}, f_{7}(t) \in\left\{T_{1}, T_{2}, T_{3}, T_{4}, T_{5}, T_{6}\right\}$,

and $f_{7}\left(t_{6}\right)=T_{6} \exists t_{6} \in \bar{T}_{6} \backslash \bar{T}_{7}$,

$f_{8}: X \backslash \bar{T}_{8} \rightarrow Q, f_{8 \alpha}(t) \in Q$.

Then $\beta=\bigcup_{x \in X}(\{x\} \times f(x)) \in B_{X}(D)$ is regular and $\varphi$ is complete $\beta$-isomorphism

$\theta$. So $\beta \in R_{\varphi}\left(Q, D^{\prime}\right)$.

Proof. First we see that $V(D, \beta)=Q$. Considering $V(D, \beta)=\{Y \beta \mid Y \in D\}$, the properties of $f$ mapping, $\bar{T}_{i} \beta=\bigcup_{x \in \bar{T}_{i}} x \beta$ and $D^{\prime} \subseteq D$, we get

$$
\begin{aligned}
& T_{1} \in Q \Rightarrow \bar{T}_{1} \beta=T_{1} \Rightarrow T_{1} \in V(D, \beta), \\
& T_{2} \in Q \Rightarrow \bar{T}_{2} \beta=T_{1} \cup T_{2}=T_{2} \Rightarrow T_{2} \in V(D, \beta), \\
& T_{3} \in Q \Rightarrow \bar{T}_{3} \beta=T_{1} \cup T_{2} \cup T_{3}=T_{3} \Rightarrow T_{3} \in V(D, \beta), \\
& T_{4} \in Q \Rightarrow \bar{T}_{4} \beta=T_{1} \cup T_{2} \cup T_{3} \cup T_{4} \cup T_{5}=T_{4} \Rightarrow T_{4} \in V(D, \beta), \\
& T_{5} \in Q \Rightarrow \bar{T}_{5} \beta=\left(\bar{T}_{3} \cup \bar{T}_{4}\right) \beta=\bar{T}_{3} \beta \cup \bar{T}_{4} \beta=T_{3} \cup T_{4}=T_{5} \Rightarrow T_{5} \in V(D, \beta), \\
& T_{6} \in Q \Rightarrow \bar{T}_{6} \beta=T_{1} \cup T_{2} \cup T_{3} \cup T_{4} \cup T_{5} \cup T_{6}=T_{6} \Rightarrow T_{6} \in V(D, \beta), \\
& T_{7} \in Q \Rightarrow \bar{T}_{7} \beta=T_{1} \cup T_{2} \cup T_{3} \cup T_{4} \cup T_{5} \cup T_{7}=T_{7} \Rightarrow T_{7} \in V(D, \beta), \\
& T_{8} \in Q \Rightarrow \bar{T}_{8} \beta=\left(\bar{T}_{6} \cup \bar{T}_{7}\right) \beta=\bar{T}_{6} \beta \cup \bar{T}_{7} \beta=T_{6} \cup T_{7}=T_{8} \Rightarrow T_{8} \in V(D, \beta) .
\end{aligned}
$$

Then $Q \subseteq V(D, \beta)$. Also,

$$
\begin{aligned}
Z \in V(D, \beta) & \Rightarrow Z=Y \beta, \exists Y \in D \\
& \Rightarrow Z=Y \beta=\bigcup_{y \in Y} y \beta=\bigcup_{y \in Y} f(y) \in Q
\end{aligned}
$$

since $f(y) \in Q$ and $Q$ is closed set-theoretic union. Therefore, $V(D, \beta) \subseteq Q$. Hence $V(D, \beta)=Q$.

Also, $\beta=\bigcup_{T \in V\left(X^{*}, \beta\right)}\left(Y_{T}^{\beta} \times T\right)$ is quasinormal representation of $\beta$ since $\emptyset \notin Q$. From the definition of $\beta, f(x)=x \beta$ for all $x \in X$. It is easily seen that 
$V\left(X^{*}, \beta\right)=V(D, \beta)=Q$. We get $\beta=\bigcup_{i=1}^{8}\left(Y_{i}^{\beta} \times T_{i}\right)$.

On the other hand

$$
\begin{aligned}
& t \in \bar{T}_{1} \Rightarrow t \beta=f(t)=T_{1} \Rightarrow t \in Y_{1}^{\beta} \Rightarrow \bar{T}_{1} \subseteq Y_{1}^{\beta}, \\
& t \in \bar{T}_{2}=\bar{T}_{1} \cup\left(\left(\bar{T}_{3} \cap \bar{T}_{4}\right) \backslash \bar{T}_{1}\right) \Rightarrow t \beta=f(t) \in\left\{T_{1}, T_{2}\right\} \Rightarrow t \in Y_{1}^{\beta} \cup Y_{2}^{\beta} \\
& \Rightarrow \bar{T}_{2} \subseteq Y_{1}^{\beta} \cup Y_{2}^{\beta} \\
& t \in \bar{T}_{3}=\bar{T}_{1} \cup\left(\left(\bar{T}_{3} \cap \bar{T}_{4}\right) \backslash \bar{T}_{1}\right) \cup\left(\bar{T}_{3} \backslash \bar{T}_{4}\right) \Rightarrow t \beta=f(t) \in\left\{T_{1}, T_{2}, T_{3}\right\} \\
& \Rightarrow t \in Y_{1}^{\beta} \cup Y_{2}^{\beta} \cup Y_{3}^{\beta} \\
& \Rightarrow \bar{T}_{4} \subseteq Y_{1}^{\beta} \cup Y_{2}^{\beta} \cup Y_{3}^{\beta}, \\
& t \in \bar{T}_{4}=\bar{T}_{1} \cup\left(\left(\bar{T}_{3} \cap \bar{T}_{4}\right) \backslash \bar{T}_{1}\right) \cup\left(\bar{T}_{4} \backslash \bar{T}_{3}\right) \Rightarrow t \beta=f(t) \in\left\{T_{1}, T_{2}, T_{4}\right\} \\
& \Rightarrow t \in Y_{1}^{\beta} \cup Y_{2}^{\beta} \cup Y_{4}^{\beta} \\
& \Rightarrow \bar{T}_{4} \subseteq Y_{1}^{\beta} \cup Y_{2}^{\beta} \cup Y_{4}^{\beta} \text {, } \\
& t \in \bar{T}_{6}=\left(\bar{T}_{6} \backslash \bar{T}_{7}\right) \cup\left(\left(\bar{T}_{7} \cap \bar{T}_{6}\right) \backslash \bar{T}_{5}\right) \cup \bar{T}_{3} \cup \bar{T}_{4} \\
& \Rightarrow t \beta=f(t) \in\left\{T_{1}, T_{2}, T_{3}, T_{4}, T_{5}, T_{6}\right\} \\
& \Rightarrow t \in Y_{1}^{\beta} \cup Y_{2}^{\beta} \cup Y_{3}^{\beta} \cup Y_{4}^{\beta} \cup Y_{5}^{\beta} \cup Y_{6}^{\beta} \\
& \Rightarrow \bar{T}_{6} \subseteq Y_{1}^{\beta} \cup Y_{2}^{\beta} \cup Y_{3}^{\beta} \cup Y_{4}^{\beta} \cup Y_{5}^{\beta} \cup Y_{6}^{\beta} \text {, } \\
& t \in \bar{T}_{7}=\left(\bar{T}_{7} \backslash \bar{T}_{6}\right) \cup\left(\left(\bar{T}_{7} \cap \bar{T}_{6}\right) \backslash \bar{T}_{5}\right) \cup \bar{T}_{3} \cup \bar{T}_{4} \\
& \Rightarrow t \beta=f(t) \in\left\{T_{1}, T_{2}, T_{3}, T_{4}, T_{5}, T_{7}\right\} \\
& \Rightarrow t \in Y_{1}^{\beta} \cup Y_{2}^{\beta} \cup Y_{3}^{\beta} \cup Y_{4}^{\beta} \cup Y_{5}^{\beta} \cup Y_{7}^{\beta} \\
& \Rightarrow \bar{T}_{6} \subseteq Y_{1}^{\beta} \cup Y_{2}^{\beta} \cup Y_{3}^{\beta} \cup Y_{4}^{\beta} \cup Y_{5}^{\beta} \cup Y_{7}^{\beta} \text {, }
\end{aligned}
$$

Also, by using $f_{2}\left(t_{2}\right)=T_{2}, \exists t_{2} \in \bar{T}_{2} \backslash \bar{T}_{1}$, we obtain $Y_{2}^{\beta} \cap \bar{T}_{2} \neq \emptyset$. Similarly, from properties of $f_{3}, f_{4}, f_{6}, f_{7}$, be seen $Y_{3}^{\beta} \cap \bar{T}_{3} \neq \emptyset, Y_{4}^{\beta} \cap \bar{T}_{4} \neq \emptyset, Y_{6}^{\beta} \cap \bar{T}_{6} \neq \emptyset$ and $Y_{7}^{\beta} \cap \bar{T}_{7} \neq \emptyset$. Therefore the mapping $\varphi: Q \rightarrow D^{\prime}=\left\{\bar{T}_{1}, \bar{T}_{2}, \ldots, \bar{T}_{8}\right\}$ to be defined $\varphi\left(T_{i}\right)=\bar{T}_{i}$ satisfy the conditions in (3.9) for $\beta$. Hence $\varphi$ is complete $\beta$-isomorhism because of $\varphi(T) \beta=\bar{T} \beta=T$, for all $T \in V(D, \beta)$. By Theorem $3.4, \beta \in R_{\varphi}\left(Q, D^{\prime}\right)$.

Therefore, there is one to one correspondence between the elements of $R_{\varphi}\left(Q, D^{\prime}\right)$ and the set of ordered systems of disjoint mappings.

Theorem 3.7. Let $X$ be a finite set and $Q$ be $X I$ - semilattice. If

$$
D^{\prime}=\left\{\bar{T}_{1}, \bar{T}_{2}, \bar{T}_{3}, \bar{T}_{4}, \bar{T}_{5}, \bar{T}_{6}, \bar{T}_{7}, \bar{T}_{8}\right\}
$$

is $\alpha$ - isomorphic to $Q$ and $\Omega(Q)=m_{0}$, then

$$
\left|R\left(D^{\prime}\right)\right|=m_{0} \cdot 4 \cdot\left(2^{\left.\mid \bar{T}_{3} \cap \bar{T}_{4}\right) \backslash \bar{T}_{2} \mid}\left(2^{\left|\bar{T}_{2} \backslash \bar{T}_{1}\right|}-1\right)\right) \cdot\left(3^{\left|\bar{T}_{4} \backslash \bar{T}_{3}\right|}-2^{\left|\bar{T}_{4} \backslash \bar{T}_{3}\right|}\right)
$$




$$
\begin{aligned}
& \cdot\left(3^{\left|\bar{T}_{3} \backslash \bar{T}_{4}\right|}-2^{\left|\bar{T}_{3} \backslash \bar{T}_{4}\right|}\right) \cdot 5^{\left|\left(\bar{T}_{7} \cap \bar{T}_{6}\right) \backslash \bar{T}_{5}\right|} \cdot\left(6^{\left|\bar{T}_{7} \backslash \bar{T}_{6}\right|}-5^{\left|\bar{T}_{7} \backslash \bar{T}_{6}\right|}\right) \\
& \cdot\left(6^{\left|\bar{T}_{6} \backslash \bar{T}_{7}\right|}-5^{\left|\bar{T}_{6} \backslash \bar{T}_{7}\right|}\right) \cdot 8^{\left|X \backslash \bar{T}_{8}\right|}
\end{aligned}
$$

Proof. Lemma 3.5 and Lemma 3.6 show us that the number of the ordered system of disjoint mappings $\left(f_{1 \alpha}, f_{2 \alpha}, f_{3 \alpha}, f_{4 \alpha}, f_{5 \alpha}, f_{6 \alpha}, f_{7 \alpha}, f_{8 \alpha}\right)$ is equal to $\left|R_{\varphi}\left(Q, D^{\prime}\right)\right|$, which $\alpha \in B_{X}(D)$ regular element, $V(D, \alpha)=Q$ and $\varphi: Q \rightarrow D^{\prime}$ is a complete $\alpha$-isomorphism.

From the Theorem 2.2, the number of the mappings $f_{1 \alpha}, f_{2 \alpha}, f_{3 \alpha}, f_{4 \alpha}, f_{5 \alpha}$, $f_{6 \alpha}, f_{7 \alpha}$ and $f_{8 \alpha}$ are respectively as

$$
\begin{aligned}
& 1,\left(2^{\left|\left(\bar{T}_{3} \cap \bar{T}_{4}\right) \backslash \bar{T}_{2}\right|}\left(2^{\left|\bar{T}_{2} \backslash \bar{T}_{1}\right|}-1\right)\right),\left(3^{\left|\bar{T}_{4} \backslash \bar{T}_{3}\right|}-2^{\left|\bar{T}_{4} \backslash \bar{T}_{3}\right|}\right),\left(3^{\left|\bar{T}_{3} \backslash \bar{T}_{4}\right|}-2^{\left|\bar{T}_{3} \backslash \bar{T}_{4}\right|}\right), \\
& 5^{\left|\left(\bar{T}_{7} \cap \bar{T}_{6}\right) \backslash \bar{T}_{5}\right|},\left(6^{\left|\bar{T}_{7} \backslash \bar{T}_{6}\right|}-5^{\left|\bar{T}_{7} \backslash \bar{T}_{6}\right|}\right),\left(6^{\left|\bar{T}_{6} \backslash \bar{T}_{7}\right|}-5^{\left|\bar{T}_{6} \backslash \bar{T}_{7}\right|}\right), 8^{\left|X \backslash \bar{T}_{8}\right|} .
\end{aligned}
$$

Now, we determine the number of regular elements

$$
\begin{aligned}
\left|R_{\varphi}\left(Q, D^{\prime}\right)\right| & =\left(2^{\left.\mid \bar{T}_{3} \cap \bar{T}_{4}\right) \backslash \bar{T}_{2} \mid}\left(2^{\left|\bar{T}_{2} \backslash \bar{T}_{1}\right|}-1\right)\right) \cdot\left(3^{\left|\bar{T}_{4} \backslash \bar{T}_{3}\right|}-2^{\left|\bar{T}_{4} \backslash \bar{T}_{3}\right|}\right) \\
& \cdot\left(3^{\left|\bar{T}_{3} \backslash \bar{T}_{4}\right|}-2^{\left|\bar{T}_{3} \backslash \bar{T}_{4}\right|}\right) \cdot 5^{\left.\mid \bar{T}_{7} \cap \bar{T}_{6}\right) \backslash \bar{T}_{5} \mid} \cdot\left(6^{\left|\bar{T}_{7} \backslash \bar{T}_{6}\right|}-5^{\left|\bar{T}_{7} \backslash \bar{T}_{6}\right|}\right) \\
& \cdot\left(6^{\left|\bar{T}_{6} \backslash \bar{T}_{7}\right|}-5^{\left|\bar{T}_{6} \backslash \bar{T}_{7}\right|}\right) \cdot 8^{\left|X \backslash \bar{T}_{8}\right|}
\end{aligned}
$$

The number of all automorphisms of the semilattice $Q$ is $q=4$. These are

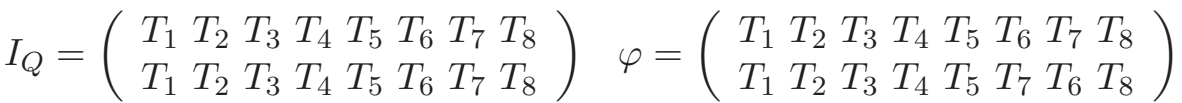

$$
\begin{aligned}
& \theta=\left(\begin{array}{llllllll}
T_{1} & T_{2} & T_{3} & T_{4} & T_{5} & T_{6} & T_{7} & T_{8} \\
T_{1} & T_{2} & T_{4} & T_{3} & T_{5} & T_{7} & T_{6} & T_{8}
\end{array}\right) \quad \tau=\left(\begin{array}{cccccccc}
T_{1} & T_{2} & T_{3} & T_{4} & T_{5} & T_{6} & T_{7} & T_{8} \\
T_{1} & T_{2} & T_{4} & T_{3} & T_{5} & T_{6} & T_{7} & T_{8}
\end{array}\right) .
\end{aligned}
$$

Therefore by using Theorem 2.3,

$$
\begin{aligned}
\left|R\left(D^{\prime}\right)\right| & =m_{0} \cdot 4 \cdot\left(2^{\left|\left(\bar{T}_{3} \cap \bar{T}_{4}\right) \backslash \bar{T}_{2}\right|}\left(2^{\left|\bar{T}_{2} \backslash \bar{T}_{1}\right|}-1\right)\right) \cdot\left(3^{\left|\bar{T}_{4} \backslash \bar{T}_{3}\right|}-2^{\left|\bar{T}_{4} \backslash \bar{T}_{3}\right|}\right) \\
& \cdot\left(3^{\left|\bar{T}_{3} \backslash \bar{T}_{4}\right|}-2^{\left|\bar{T}_{3} \backslash \bar{T}_{4}\right|}\right) \cdot 5^{\mid\left(\bar{T}_{7} \cap \bar{T}_{6} \backslash \backslash \bar{T}_{5} \mid\right.} \cdot\left(6^{\left|\bar{T}_{7} \backslash \bar{T}_{6}\right|}-5^{\left|\bar{T}_{7} \backslash \bar{T}_{6}\right|}\right) \\
& \cdot\left(6^{\left|\bar{T}_{6} \backslash \bar{T}_{7}\right|}-5^{\left|\bar{T}_{6} \backslash \bar{T}_{7}\right|}\right) \cdot 8^{\left|X \backslash \bar{T}_{8}\right|}
\end{aligned}
$$

is obtained.

Example 1. Let $X=\{1,2,3,4,5,6\}$ and

$$
\begin{aligned}
D= & \left\{T_{1}=\{1\}, T_{2}=\{1,2\}, T_{3}=\{1,2,3\}, T_{4}=\{1,2,4\}, T_{5}=\{1,2,3,4\}\right. \\
& \left.T_{6}=\{1,2,3,4,5\}, T_{7}=\{1,2,3,4,6\}, T_{8}=\{1,2,3,4,5,6\}\right\} .
\end{aligned}
$$


$D$ is an $X$-semilattice of unions since $D$ is closed the union of sets. Moreover $D$ satisfies the conditions in (3.1). Then, $D$ is an $X I$-semilattice. Let $D=Q$. Therefore $|\Omega(Q)|=1$. Besides, the number of all automorphisms of $Q$ is $q=4$. By using Theorem 3.7

$$
\begin{aligned}
|R(Q)| & =1 \cdot 4 \cdot\left(2^{\left.\mid \bar{T}_{3} \cap \bar{T}_{4}\right) \backslash \bar{T}_{2} \mid}\left(2^{\left|\bar{T}_{2} \backslash \bar{T}_{1}\right|}-1\right)\right) \cdot\left(3^{\left|\bar{T}_{4} \backslash \bar{T}_{3}\right|}-2^{\left|\bar{T}_{4} \backslash \bar{T}_{3}\right|}\right) \\
& \cdot\left(3^{\left|\bar{T}_{3} \backslash \bar{T}_{4}\right|}-2^{\left|\bar{T}_{3} \backslash \bar{T}_{4}\right|}\right) \cdot 5^{\left|\left(\bar{T}_{7} \cap \bar{T}_{6}\right) \backslash \bar{T}_{5}\right|} \cdot\left(6^{\left|T_{7} \backslash \bar{T}_{6}\right|}-5^{\left|\bar{T}_{7} \backslash \bar{T}_{6}\right|}\right) \\
& \cdot\left(6^{\left|\bar{T}_{6} \backslash \bar{T}_{7}\right|}-5^{\left|\bar{T}_{6} \backslash \bar{T}_{7}\right|}\right) \cdot 8^{\left|X \backslash \bar{T}_{8}\right|} \\
& =4
\end{aligned}
$$

is obtained.

\section{References}

[1] Schröder, E., Vorlesungen über die Algebra der Logik, 3 vols. Leipzig: B.G. Teubner. Reprints: (1966), Chelsea; (2000), Thoemmes Press.

[2] A. N. Whitehead and B. Russell, Principia mathematica, vol. 1, Cambridge University Press, (1925).

[3] Riguet, Relations binaires, fermetures, correspondances de Galois, Buletin de Socit Mathematique de France, vol. 79 (1948), 114-155.

[4] Riguet, Quelques propriety's des relations difonctionnelles, Comptes renlus heb- modaires des seances de Academie des Sciences, vol. 230 (1950), 19992000 .

[5] V. V. Vagner, The theory of relations and the algebra of partial transformations, Theory of Semigroups and Its Applications, Saratov, No. 1 (1965), 3-178.

[6] Diasamidze Ya., Complete Semigroups of Binary Relations. Journal of Mathematical Sciences, Plenum Publ. Cor., New York, Vol. 117, No. 4 (2003), 4271-4319.

[7] Diasamidze Ya., Makharadze Sh., Complete semigroups of binary relations. Sputnik +, Moscow (2010), (in Russian).

[8] Diasamidze Ya., Makharadze Sh., Partenadze G., Givradze O., On finite $X$ - semilattices of unions. Journal of Mathematical Sciences, Plenum Publ. Cor., New York, Vol. 141, No. 4 (2007), 1134-1181. 
[9] Diasamidze Ya., Makharadze Sh., Complete Semigroups of Binary Relations Defined by $X$ - Semilattices of Unions. Journal of Mathematical Sciences, Plenum Publ. Cor., New York, Vol. 166, No. 5 (2010), 615-633. 